\title{
6.78MHz Capacitive Coupling Wireless Power Transfer System
}

\author{
Kang Hyun $\mathrm{Yi}^{\dagger}$ \\ ${ }^{\dagger}$ School of Electrical and Electric Engineering, Daegu University, Gyeongsan, Korea
}

\begin{abstract}
Wireless power transfer technologies typically include inductive coupling, magnetic resonance, and capacitive coupling methods. Among these methods, capacitive coupling wireless power transfer (CCWPT) has been studied to overcome the drawbacks of other approaches. CCWPT has many advantages such as having a simple structure, low standing power loss, reduced electromagnetic interference (EMI) and the ability to transfer power through metal barriers. In this paper, the CCWPT system with $6.78 \mathrm{MHz}$ class $\mathrm{D}$ inverter is proposed and analyzed. The proposed system consists of a $6.78 \mathrm{MHz}$ class $\mathrm{D}$ inverter with a LC low pass filter, capacitor between a transmitter and a receiver, and impedance transformers. The system is verified with a prototype for charging mobile devices.
\end{abstract}

Key words: 6.78Mz, Capacitive Coupling Power Transfer, Class D inverter, Wireless Power Transfer

\section{INTRODUCTION}

Recently, the application of wireless power transfer (WPT) in charging mobile devices and the electric vehicles is widely researched. WPT are generally classified as magnetic resonance, inductive coupling, and capacitive coupling. Magnetic resonance WPT uses resonance with the transmitting coil and receiving coil. The method has many advantages such as long distance transfer and user convenience. However, the system has not been commercialized because of electromagnetic interference (EMI), human activity, and some imposed regulations [1], [2]. Inductive coupling WPT follows the same principle of a transformer in the power converter. Despite having good efficiency and developed commercialization techniques, inductive coupling WPT has disadvantages such as one position power transfer, heat dissipation in the metal barrier, and the large coil volume [3], [4]. The prior two approaches employ the magnetic field, hence the presence of radiation noise and power transfer metal interference. In contrast, capacitive coupling wireless power transfer (CCWPT) uses electric field and displacement current to transfer wirelessly power; this approach is also called contactless power

Manuscript received Jul. 29, 2014; accepted Feb. 26, 2015

Recommended for publication by Associate Editor Hao Ma.

†Corresponding Author: khyi@daegu.ac.kr

Tel: +82-53-850-6652, Fax: +82-53-850-6619, Daegu University

School of Electronic and Electrical Eng., Daegu University, Korea

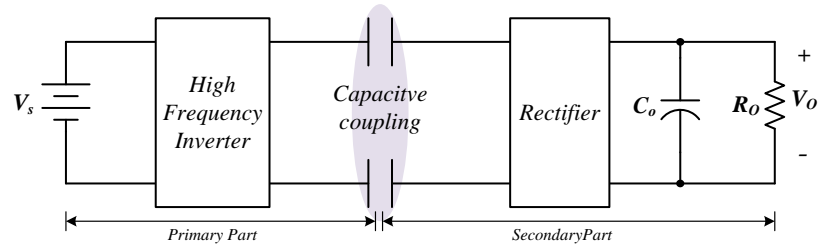

Fig. 1. The simple structure of the CPT system.

transfer [5], [6].

Fig. 1 shows the simple structure of the CCWPT system. Direct current (DC) voltage is converted to alternating current (AC) voltage that supplies two primary metal plates using a high-frequency inverter. Two secondary plates are located adjacent the structure and the electric field enables the displacement current to flow. The DC output voltage can be obtained with a rectifier. Coupling capacitance is an important factor in transferring energy and operation stability. However, the realized capacitance is limited by the available area of the device being used. The power conversion circuit is a series resonant type circuit that produces high frequency AC voltage. In the other approaches, a simple full bridge inverter is used to make the series resonance between the inductor and coupling capacitor [7]. If the obtained capacitance is very small, the simple resonant circuit limits the transferring. The quality factor is very high hence the operation is unstable.

A high-frequency operation and impedance transformation is a viable solution to overcome this drawback. Operation 


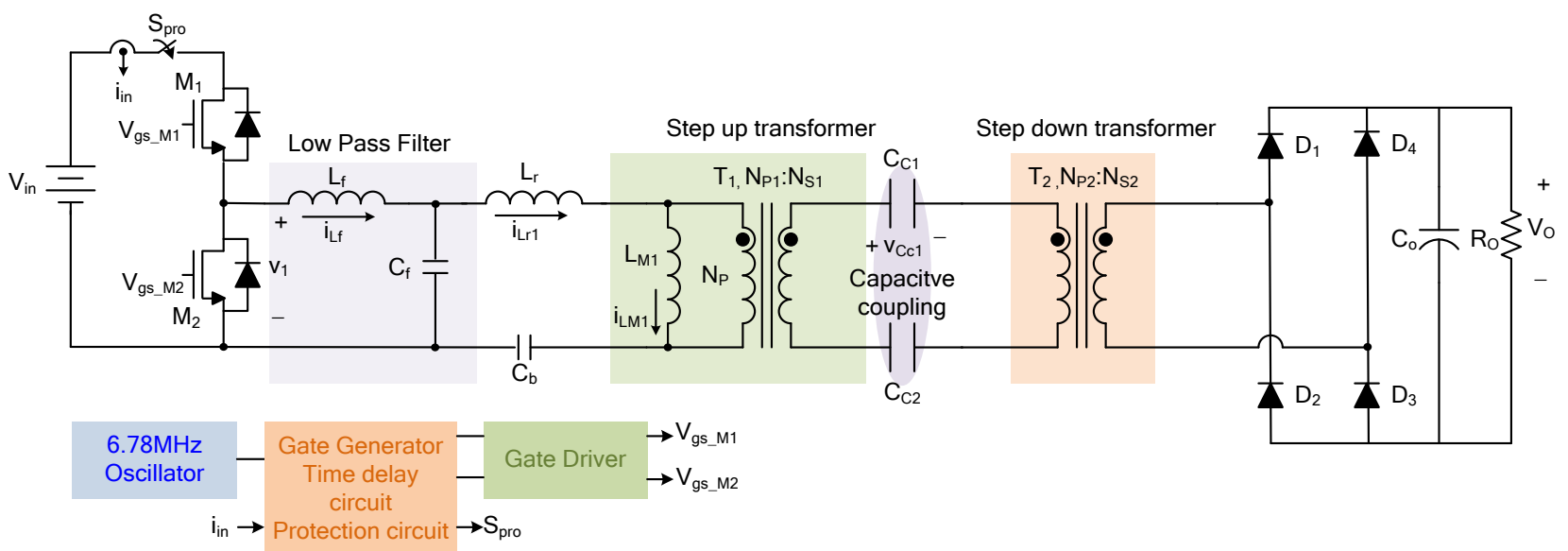

Fig. 2. A proposed 6.78 MHz CCWPT system.

frequency selection is necessary to meet wireless regulations in mobile wireless charger applications and standardize the operation frequency of industrial, scientific, and medical (ISM) band. A silicon power semiconductor cannot be driven with a very high frequency like the $\mathrm{GHz}$ operation. $6.78 \mathrm{MHz}$ and $13.56 \mathrm{MHz}$ ISM bands can drive the silicon power semiconductor, reducing the quality factor and meeting regulations. However, the $13.56 \mathrm{MHz}$ band is already used in other application. Hence $6.78 \mathrm{MHz}$ operation can meet wireless regulations without obstructing other devices and help transfer energy with a small capacitance in the CCWPT.

This paper is proposes a $6.78 \mathrm{MHz}$ CCWPT system with a Class D inverter, LC low pass filter, and impedance transformation circuit. A theoretical estimation of the capacitance is shown according to width of the plastic molding. Moreover, the design procedures of the LC filter and impedance transformation circuit are shown with the area of the electro metal plates and receiving power. The system is verified with the $5 \mathrm{~V}$ and $800 \mathrm{~mA}$ output for charging a mobile device.

\section{A PROPOSED SYSTEM}

Fig. 2 shows a proposed $6.78 \mathrm{MHz}$ CCWPT system. $S_{\text {pro }}$ is a path switch that controls the input power by monitoring input current. Class D inverter and LC low pass filter are used for the transmitting circuit. The LC low pass filter suppresses EMI noise from the rectifier. A zero voltage switching (ZVS) can be achieved by below resonance operation of the $L_{f}$ and $C_{f} . L_{r}$ is the transferring power to the receiver by a resonance with coupling capacitors, $C_{c 1}$ and $C_{c 2}$. $C_{b}$ is a DC blocking capacitor for non-saturation of a step-up transformer. A step-up transformer is used to make the impedance of the coupling capacitance, $C_{c 1}$ and $C_{c 2}$, whereas the small and step-down transformer reduces the quality-factor (Q-factor) of the series resonant circuit. The optimized Q-factor can be obtained by turn-ratios of the step-up and step-down transformers. Fig. 3 shows the waveforms of the resonance

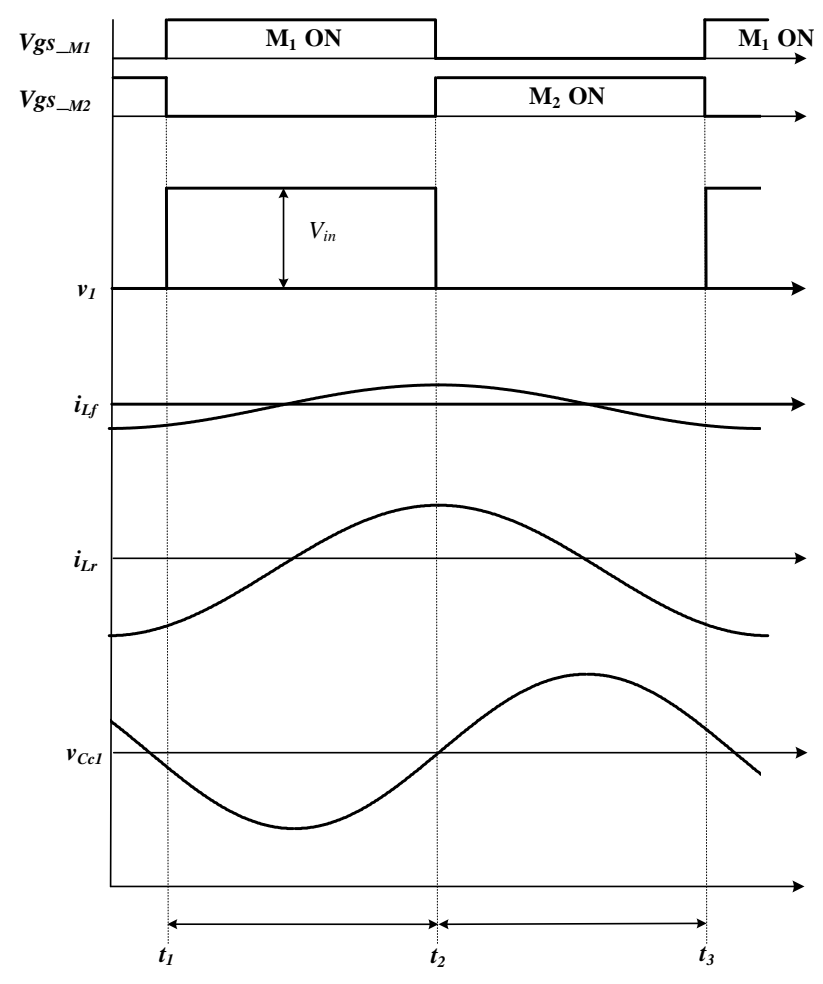

Fig. 3. Waveforms of the proposed system.

parts. The resonance with $L_{r}, C_{c 1}$, and $C_{c 2}$ facilitates the energy transfer to the load. A driving stage consists of a $6.78 \mathrm{MHz}$ crystal oscillator, gate signal generator, time delay circuit, protection circuit, and gate driver. The proposed system works with high frequency, hence the time delay circuit design and gate driver are important. The driving block is applied with a general circuit.

\section{DESIGN CONSIDERATIONS}

\section{A. Capacitance Estimation}

Fig. 4 shows the simple structure of a capacitor in the transmitter and receiver. The capacitor can be made with 


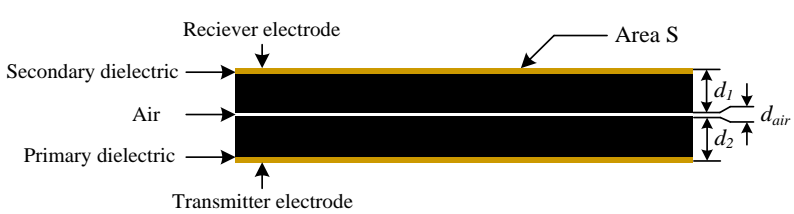

Fig. 4. Structure of capacitor between transmitter and receiver.

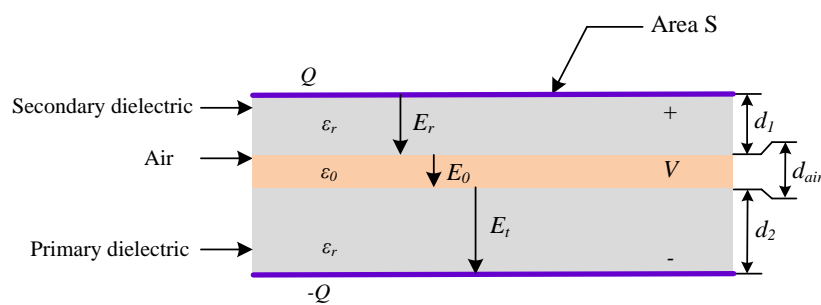

Fig. 5. Capacitance estimation by the Gauss’ law.

electrodes and dielectric layers in the transmitter, receiver, and air. The capacitance can be derived using the Gauss' law, as shown in Fig. 5 [8]. Voltage, $V$, across the capacitor and capacitance can be expressed in the Gauss's law, as follows

$$
\begin{aligned}
V & =E_{r} d_{1}+E_{0} d_{\text {air }}+E_{t} d_{2} \\
& =\frac{Q / S}{\varepsilon_{r} \varepsilon_{0}} d_{1}+\frac{Q / S}{\varepsilon_{0}} d_{\text {air }}+\frac{Q / S}{\varepsilon_{r} \varepsilon_{0}} d_{2} \\
C & =\frac{Q}{V}=\frac{\varepsilon_{0} S}{\left(d_{1}+d_{2}\right) / \varepsilon_{r}+d_{\text {air }}}
\end{aligned}
$$

where $S$ is the area of the electrode, $E_{r}$ is the electric field in the receiving substrate, $E_{0}$ is the electric field in the air part, $E_{t}$ is the electric field in the transmitting substrate, $\varepsilon_{0}$ and $\varepsilon_{\mathrm{r}}$ $\varepsilon_{0}$ are the permittivity of the air and dielectric substrate, and $d_{1}, d_{\text {air }}$, and $d_{2}$ are the widths. The capacitance is critically determined by the width of the transmitting and receiving dielectric layer. The capacitance can be obtained according to the width of the dielectric layer if the dielectric layer is the acrylonitrile butadiene styrene (ABS) resin, the relative permittivity of the ABS resin is about 4 , the $d_{0}$ is $0.00008 \mathrm{~m}$, and the area of the electrode is $0.004 \mathrm{~m}^{2}$, as shown in Fig. 6. The capacitance is approximately $60 \mathrm{pF}$ in a $2 \mathrm{~mm}$-width dielectric layer.

\section{B. Low Pass Filter Design}

A low pass filter design is important for the ZVS operation of the main switches and suppression of harmonic component. Fig. 7 shows the AC equivalent circuit for the LC filter design. $R_{a c}$ is an equivalent $\mathrm{AC}$ resistance for the fundamental transfer gain for the resonant DC/DC converter and can be expressed as

$$
R_{a c}=n_{1} n_{2} \frac{8}{\pi^{2}} R_{o}
$$

where $R_{o}$ is the output resistance [9]. The impedance of the LC low pass filter can be expressed as

$$
Z_{a c}=j \omega L_{r}+\frac{1}{j \omega\left(C_{C 1}+C_{C 2}\right) / C_{C 1} C_{C 2} / n_{1}^{2}}+R_{a c}
$$

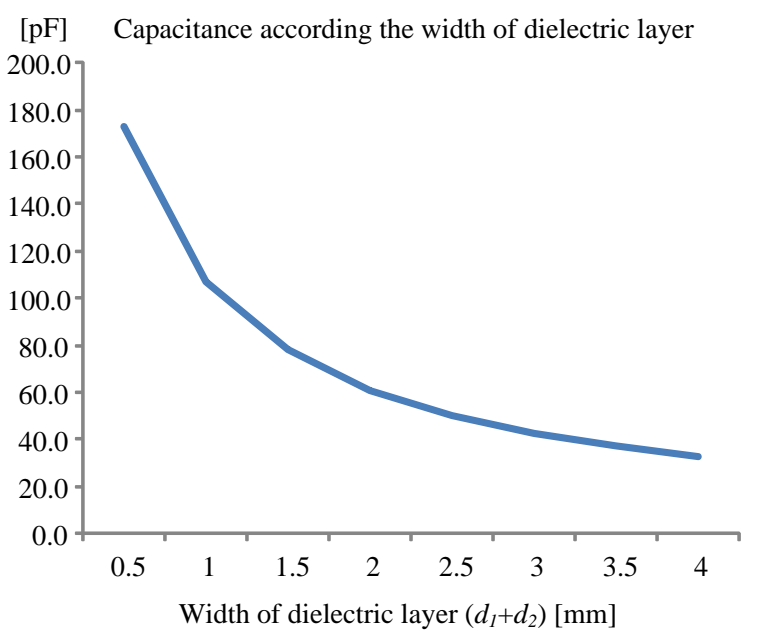

Fig. 6. Capacitance according the width of dielectric layer.

where $\omega$ is the angular frequency. The design of $L_{f}$ and $C_{f}$ is can be obtained with the Butterworth low pass filter. The Butterworth filter can be designed using equation (5) and equation (6) to determine the $L_{f}$ and $C_{f}$ values

$$
\begin{gathered}
L_{f}=\frac{\left|Z_{a c, \omega_{c}}\right| \times \sqrt{2}}{\omega_{c}} \\
C_{f}=\frac{1}{\left|Z_{a c, \omega_{c}}\right| \times \omega_{c} \times \sqrt{2}}
\end{gathered}
$$

where $\omega_{c}$ is the cutoff frequency and $\left|Z_{a c, \omega_{c}}\right|$ is the magnitude of the output impedance at the $\omega_{c}$.

C. Voltage Gain Considering the Parasitic Component of the Step-Up and Step-Down Transformer

Ideally, the fundamental voltage gain of the CCWPT is part of the resonant frequency because the CCWPT is a series resonant converter, as shown in Fig. 8. The voltage gain can be expressed as

$$
\begin{aligned}
& G_{v}=\frac{V_{R}^{F}}{V_{i n}^{F}}=\frac{1}{\sqrt{1+Q^{2}\left(\frac{f}{f_{0}}-\frac{f_{0}}{f}\right)^{2}}} \\
& Q=\frac{\sqrt{L_{r} /\left(\frac{C_{C 1}+C_{C 2}}{n_{1}^{2}\left(C_{C 1} \times C_{C 2}\right)}\right)}}{f_{0}=\frac{R_{a c}}{\sqrt{L_{r}\left(\frac{C_{C 1}+C_{C 2}}{n_{1}^{2}\left(C_{C 1} \times C_{C 2}\right)}\right)}}}
\end{aligned}
$$

where $V_{R}{ }^{F}$ and $V_{\text {in }}{ }^{F}$ are the fundamental components of the input and output, $Q$ is the quality factor, $f_{0}$ is the resonant frequency, and $f$ is the switching frequency of the class $D$ 

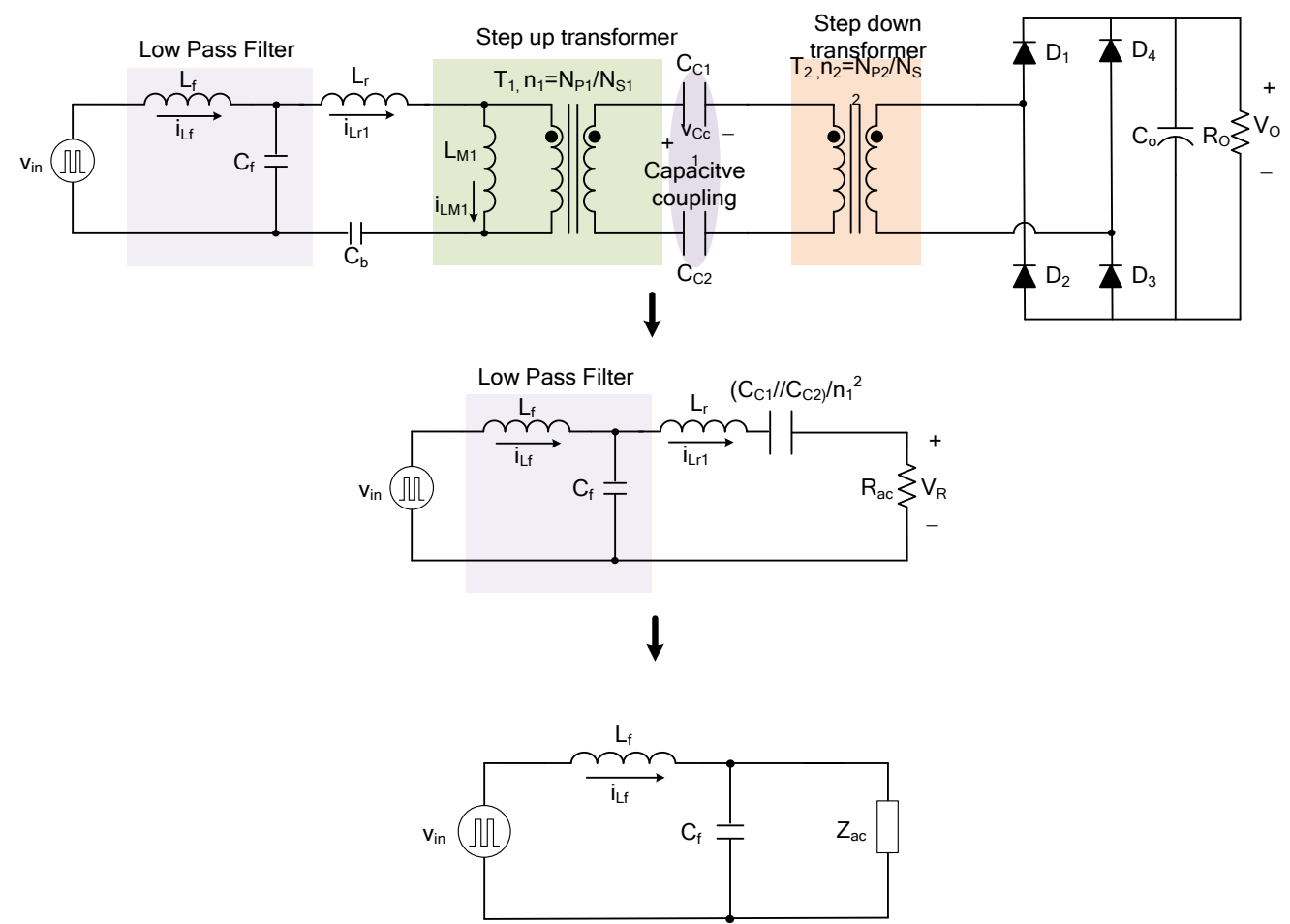

Fig. 7. AC equivalent circuit for the LC filter design.

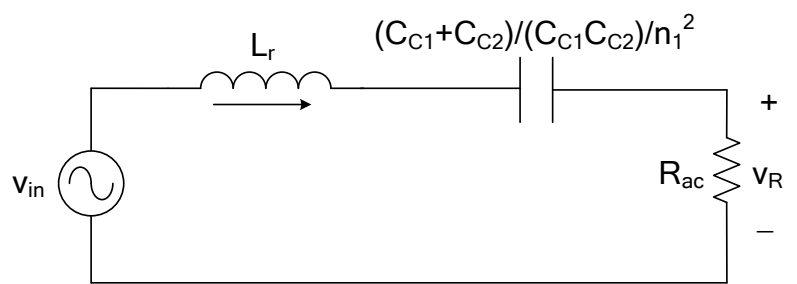

Fig. 8. Equivalent circuit for the voltage gain without the parasitic components.

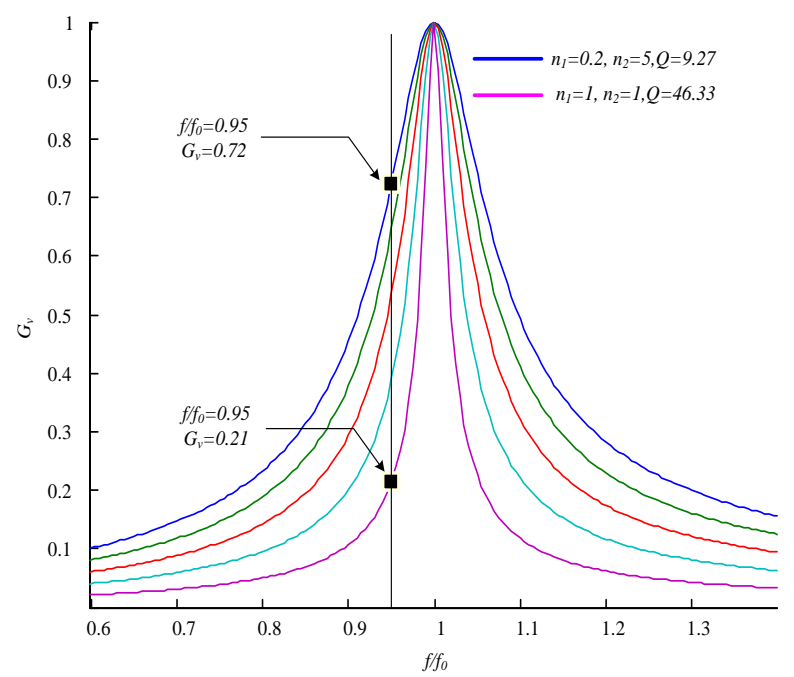

Fig. 9. Voltage gain curve without the parasitic components.

inverter. The $Q$ is high and the voltage gain curve is sharp, hence the unstable operation. The output voltage feedback cannot control the CCWPT, thus a low $Q$ is needed to obtain the constant output when the resonant frequency changes.
TABLE I

EXPERIMENT SPECIFICATION AND PARTS

\begin{tabular}{ccc}
\hline Specification/ & Part & Value \\
Components & $V_{s}$ & $10 \mathrm{~V} 16 \mathrm{~V}$ \\
\hline Boost Input & $V_{\text {in }}$ & $22 \mathrm{~V}$ \\
Boost Output & $M_{1}$ and $M_{2}$ & $F D S 3601$ \\
Inverter Switch & $L_{f}$ & $1.5 \mu \mathrm{H}$ \\
Low Pass $L$ & $C_{f}$ & $220 \mathrm{pF}$ \\
Low Pass C & $L_{r}$ & $440 \mathrm{nH}$ \\
Resonant Inductor & $N_{p 1}: N_{s 1}$ & $1: 4$ \\
Step-up Transformer & $C_{c 1}$ and & $200 p F$ \\
Coupling Capacitor & $C_{c 2}$ & $4: 1$ \\
Step-down Transformer & $N_{p 2}: N_{s 2}$ & $B S 630$ \\
Rectifier & $D_{1} \sim D_{4}$ & $2 \mu F$ \\
Output Capacitor & $C_{o}$ & $0.04 \mathrm{~mm}$ \\
Dielectric Layer Width & $d_{1}+d_{2}$ & $800 \mathrm{~mA}(5 \mathrm{~V})$ \\
Charging Current & Buck & \\
\hline
\end{tabular}

Fig. 9 shows the voltage gain according to the $n 1$ and $n 2$ if the coupling capacitance is $30 \mathrm{pF}$. The CCWPT can determine the output voltage gain with the design of the turn ratios of the step-up and step-down transformer. If the switching frequency only changes by $5 \%$, the voltage gain is sharply reduced without the step-up and step-down transformers, as shown in Fig. 9. Therefore, the impedance transformation circuit is needed to obtain the small $Q$ for a stable output voltage without feedback control.

The parasitic components will be in the step-up and step-down transformer, as shown in Fig. 10, hence a detailed analysis of the voltage gain is needed. $L_{m 1}$ and $L_{m 2}$ are the 


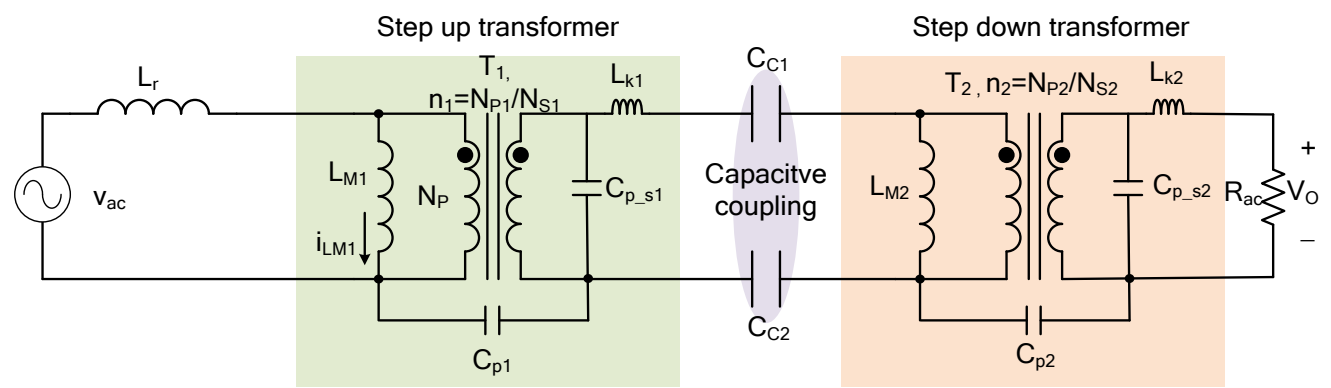

(a)

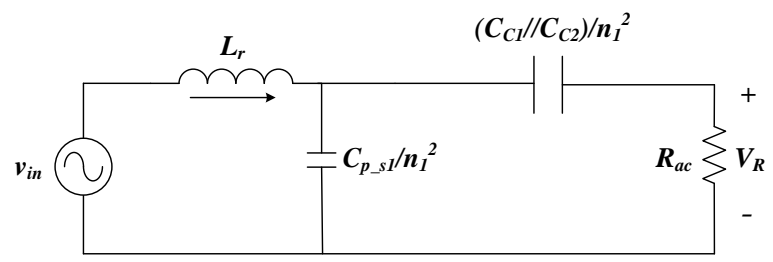

(b)

Fig. 10. Circuit diagram with the parasitic component. (a) Simplified circuit . (b) Equivalent circuit for the voltage gain.

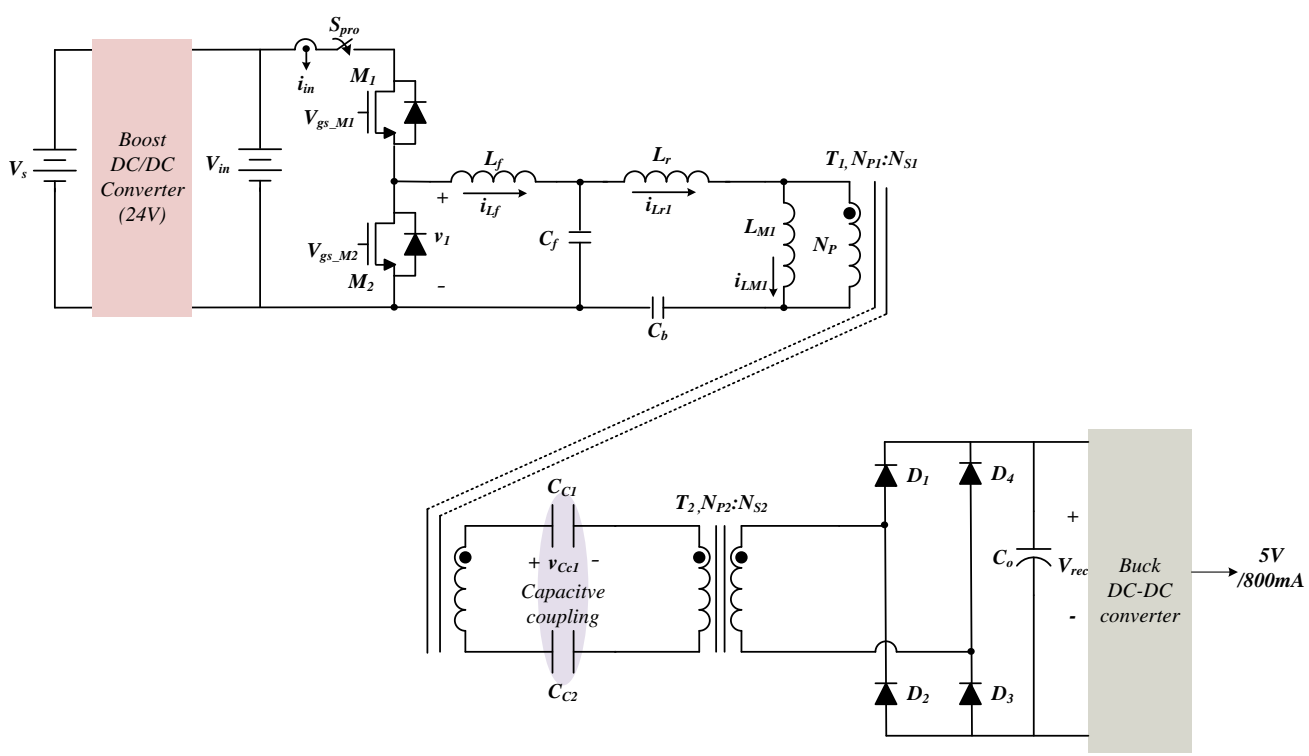

Fig. 11. Prototype system for the experiment.

magnetized inductors with a large inductance, thus these inductors cannot be considered in the voltage gain. The turn ratio of the step-up transformer is small and turn ratio of the step-down transformer is large hence a parasitic capacitor is placed between the copper wires [10]. The capacitor in the secondary step-up transformer is dominant, and the equivalent circuit for voltage gain can be obtained, as shown in Fig. 10(b). The voltage gain with the parasitic components is expressed as

$$
G_{v}=\frac{V_{R}^{F}}{V_{i n}^{F}}=\frac{1}{\sqrt{\left(\frac{2 \pi f L_{r}}{R_{a c}}\right)^{2}+\left(L_{r} \times C_{p_{-} s 1} / n_{1}^{2}+1\right)}} .
$$

This equation shows that the output voltage cannot be obtained if the $C_{p_{-} s 1}$ is large. This implies that the very small turn ratio of the step-up transformer to make a small $\mathrm{Q}$ has a large parasitic capacitor, $C_{p_{-} s 1}$, and the power is not transferred to the load.

\section{EXPERIMENTAL RESULTS}

A prototype system is implemented to verify the design. The parts used and parameters are shown in Table 1 . The turn ratio of the step-up and step-down transformers is designed to obtain the low quality factor below one, and the RF carbonyl core is used to reduce the core loss in the $6.78 \mathrm{MHz}$ operation. The prototype is made for a mobile charger for many electronic systems such as standalone, automotive, and consumer electronic devices. The prototype system has a boost converter in the input stage and buck converter for the charger in the receiver system such as a mobile device, tablet, personal computer, or other hand-held devices, as shown in 


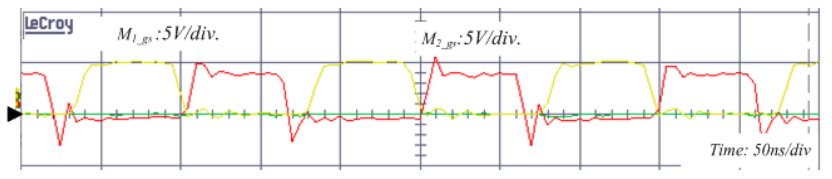

(a)

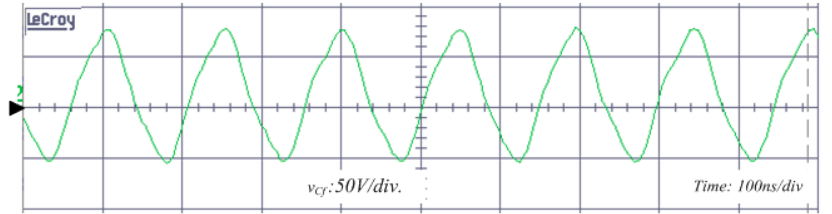

(b)

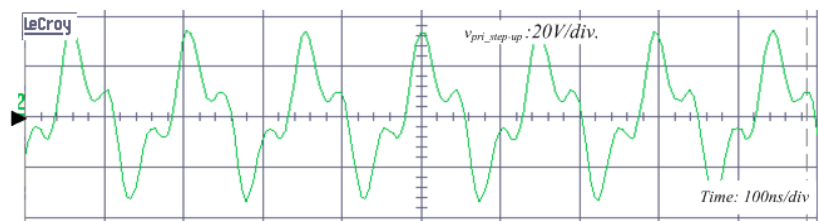

(c)

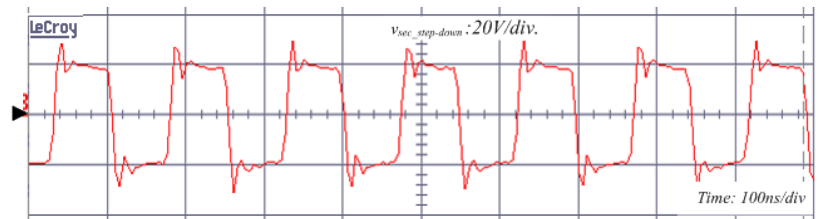

(d)

Fig. 12. Experimental waveforms. (a) Gate signals of $M_{1}$ and $M_{2}$. (b) Low Pass filter output. (c) The primary voltage of the step-up transformer. (d) The secondary voltage of the step-down transformer.

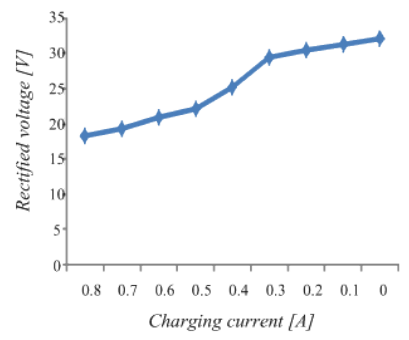

(a)

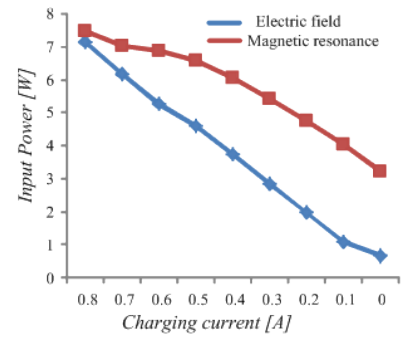

(b)
Fig. 13. Experimental results. (a) Rectified voltage according to charging current. (b) Input power according to charging current.

Fig. 11. Fig. 12 shows the experimental waveforms of the prototype CCWPT. Fig. 12(a) shows the gate signal for the Class D inverter switches. The gate signal is not simultaneously turned on. The output voltage is a $6.78 \mathrm{MHz}$ sinusoidal waveform, as shown in Fig. 12(b). The measured capacitance of the coupling capacitor is approximately 200pF with an area of $100 \mathrm{~cm}^{2}$ in the $6.78 \mathrm{MHz}$ operation. The cut-off frequency of the LC low pass filter is designed with the $9 \mathrm{MHz}$ operation, and the waveform of the LC low pass output is shown in Fig. 12(c). The excellent rectification of the received voltage is shown in Fig. 12(d). The rectified voltage is $18.6 \mathrm{~V}$ in the $5 \mathrm{~V} / 800 \mathrm{~mA}$ charging, as shown in Fig 13(a). The rectified voltage increases with decreasing charging current because the quality factor reduces with a large output voltage at the light load condition in the series resonance DC/DC converter. The $17 \mathrm{~V}$ output voltage is obtained when the position of the receiver is changed and the capacitance is $120 \mathrm{pF}$. The efficiency is about $76 \%$ from the boost output stage to the rectified stage in a full charging current. Power loss mostly occurs in semiconductor devices because of the high frequency switching loss and conduction loss in the rectifier. The magnetic resonance WPT is utilized to compare the efficiency with the proposed system. The prototype of the magnetic resonance WPT used the same 6.78MHz transmitting and receiving circuit in Fig. 11 and has two coils with impedance matching. The efficiency of the proposed CCWPT and magnetic resonance WPT are almost identical at a full charging current condition, as shown in Fig. 13(b). However, the proposed CCWPT has higher efficiency in the light load condition, and the standby power consumption is significantly less than that of the magnetic resonance WPT.

\section{CONCLUSION}

In this paper, a new CCWPT system is proposed for a mobile device charger. The system consists of a class $\mathrm{D}$ inverter, LC filter, and impedance transformation circuit such as the step-up and step-down transformers. The coupling capacitance is primarily determined by the width and permittivity of the dielectric layers. The step-up and step-down transformers increase the coupling capacitance and reduce the Q-factor, hence output power can be obtained in the resonant frequency variation. The characteristics are verified with the prototype for the $4 \mathrm{~W}$ mobile device charger, and the proposed system has better efficiency than that of the magnetic resonance WPT with full load and no load. The proposed system has a very low standby power consumption, particularly at the zero charging current. Therefore, the proposed CCWPT system can be applied in the WPT for mobile chargers and contactless power transfer for other electric devices.

\section{ACKNOWLEDGMENT}

This research was supported by the Daegu University Research Grant, 2013.

\section{REFERENCES}

[1] J. W. Kim, H. C. Son, K. H. Kim, and Y. J. Park, "Efficiency analysis of magnetic resonance wireless power transfer with intermediate resonant coil,” IEEE Antennas Wireless Propag. Lett., Vol. 10, pp. 389-392, May 2011.

[2] F. Zhang, S. A. Hackworth, W. Fu, C. Li, Z. Mao, and M. Sun, "Relay effect of wireless power transfer using strongly coupled magnetic resonances," IEEE Trans. Magn., Vol. 47, No. 5, pp. 1478-1481, May 2011. 
[3] C. S. Wang, O. H. Stielau, and G. A. Covic, "Design considerations for a contactless electric vehicle battery charger” IEEE Trans. Ind. Electron., Vol. 52, No. 5, pp. 1308-1314, Oct. 2005.

[4] J. Sallan, J. L. Villa, A. Llombart, and J. F. Sanz, “Optimal design of ICPT systems applied to electric vehicle battery charge" IEEE Trans. Ind. Electron., Vol. 56, No. 6, pp. 2140-2149, Jun. 2009

[5] A. P. Hu, C. Liu, and H. L. Li, "A novel contactless battery charging system for soccer playing robot," in Proc. 15th IEEE Int. Conf. Mechatronics and Machine Vision in Practice, pp. 646-650, 2008.

[6] A. Wahab, T. E. Chong, and L. K. Min, "Wireless pointing device using capacitive coupling," in Proc. 1997 IEEE Int. Symp. Consumer Electronics, pp. 149-152, 1997.

[7] H. Kobayashi, H. Funato and Y. Chiku, "Enhancement of transfer power of capacitive power transfer system using cascaded one pulse switching active capacitor(C-OPSAC) with three-level operation," in Power Electronics and Motion Control Conference (IPEMC), $20127^{\text {th }}$ International, pp. 884-888, 2012.

[8] H. William and H. Hayt, Engineering Electromagnetic, 5th ed., McGraw Hill, 1989.

[9] H. S. Choi, "Design consideration of half-bridge LLC resonant converter,” Journal of Power Electronics, Vol. 7, No. 1, pp. 13-20, Jan. 2007.
[10] A. K. Tripathi, K. Mainali, D. Patel, S. Bhattacharya, and K. Hatua, "Closed loop d-q control of high-voltage high-power three-phase dual active bridge converter in presence of real transformer parasitic parameters," in 2013 IEEE Energy Conversion Congress and Exposition (ECCE), pp. 5488-5495, 2013.

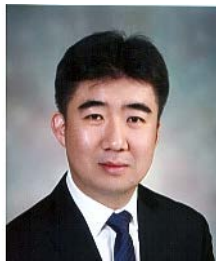

Kang-Hyun Yi was born in Korea in 1978. He received his B.S. degree in Electrical Engineering from Hanyang University, Seoul, Korea, in 2003 and M.S. and Ph.D. degrees in Electrical Engineering and Computer Science from the Korea Advanced Institute of Science and Technology, Daejeon, Korea, in 2006 and 2009, respectively. He was a Senior Engineer in Samsung Electronics Company in Suwon, Korea from 2009 to 2012. In 2012, he joined the School of Electrical and Electric Engineering, Daegu University, Gyeongsan as an Assistant Professor. His main research interests are high-efficiency DC/DC converters, soft-switching technique, digital display driver, power conversion circuit for wireless power transfer, and power electronics related to the backlight and lighting systems. Prof. Yi is a member of the Korean Institute of Power Electronics. 